MATEC Web of Conferences 12, 04020 (2014)

DOI: $10.1051 /$ matecconf $/ 20141204020$

(C) Owned by the authors, published by EDP Sciences, 2014

\title{
Role of bonding defects in a self-reinforced polypropylene (PURE) under fatigue loading
}

\author{
Christoph Skotarek and Angelika Brückner-Foit ${ }^{\mathrm{a}}$ \\ Universität Kassel, Institute for Materials Engineering, 34125 Kassel, Germany
}

\begin{abstract}
Self-reinforced plastics are manufactured by producing partly crystallized tapes, weaving fabrics, and hot-pressing of several layers of fabric. As there is no matrix accommodating the reinforcing elements the apes are locally welded to each other during hot-pressing. Upon loading pronounced strain localizations can be found using digital image correlation DIC. The localization occur on a mesoscopic scale near crossing points of the tapes and on microscopic scale between different fabric layers, if there are imperfect bondings of between the tapes. These imperfections are more likely to occur in the center of the specimen as can be visualized using computer tomography. The size and number of the bonding defects can be changed by modifying the parameters during hot pressing and by choosing different numbers of fabric layers.

The paper deals with the fatigue behavior of this type of material and the role of the bonding imperfections on the damage accumulation process. For this purpose local deformation fields using DIC determined after quasi- static loading, step-wise increased loading and cyclic loading are compared with each other. These findings are related to the damage pattern observed after final fracture.
\end{abstract}

\section{Introduction}

Micro-mechanical analysis is applied to a graded self-reinforced plastic material in order to describe the role of bonding defects under fatigue loading. As a starting point, specimens were subjected to quasistatic monotonic loading with the purpose of identifying weak points which may act as damage initiators under fatigue loading. The experiments were conducted with a tensile/compression module under a high resolution optical microscope. During each tensile test, micrographs were taken with a constant frame rate and analyzed using digital image correlation.

The focus is based on two main issues, namely the weave visible on the specimen surface and the cloth layers in depth direction.

\section{Results}

During the tensile tests, deformations on the surface were localized in the vicinity of the crossing points of the fillets (Fig. 1). It was found that the tensile strains are concentrated at the crossing points.

\footnotetext{
${ }^{a}$ Corresponding author: a.brueckner-foit@uni-kassel.de
}

This is an Open Access article distributed under the terms of the Creative Commons Attribution License 4.0, which permits unrestricted use, distribution, and reproduction in any medium, provided the original work is properly cited. 


\section{MATEC Web of Conferences}

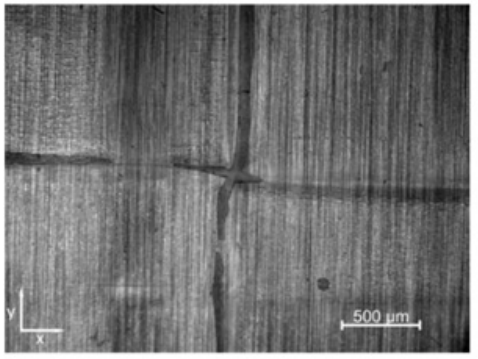

Fillet crossing point without load $\mathrm{F}$

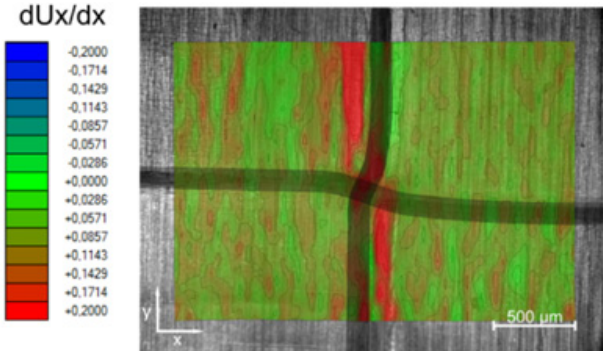

Fillet crossing point at a tensile strain of $209 \mathrm{MPa}$

fillet crossing point
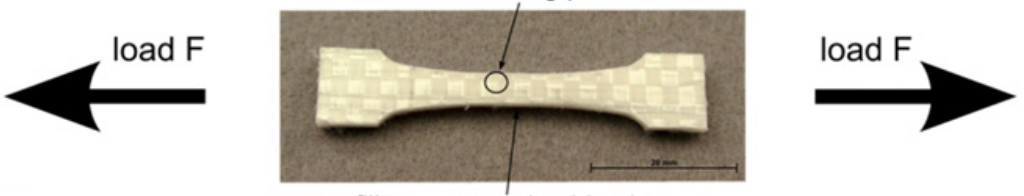

fillet structure in side view

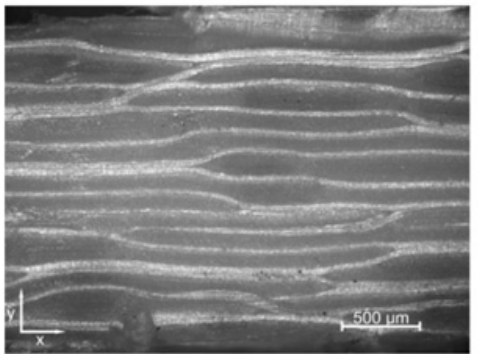

Fillet strukture in sideview without load $\mathrm{F}$

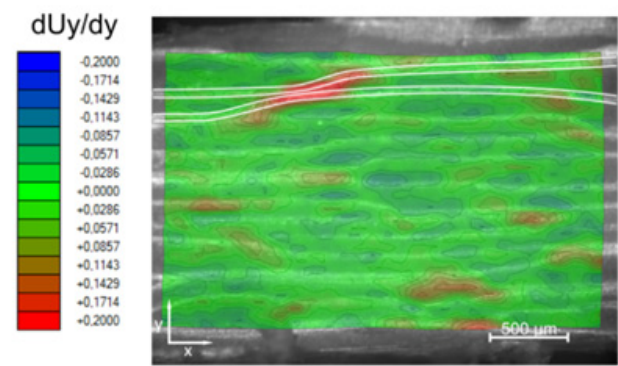

Fillet crossing point at a tensile strain of $165 \mathrm{MPa}$

Figure 1. Optical microscope: overview of some weak points and their detection via 2D-DIC.

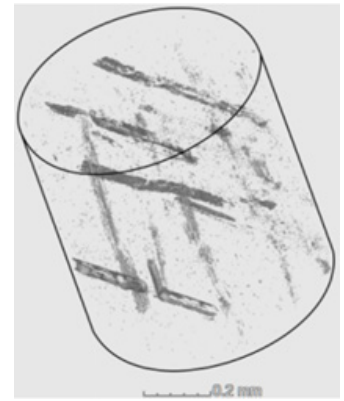

Figure 2. CT Micrograph: some regions offer different contrast structures $\rightarrow$ sign of damaged substructures in the volume. 
Moreover there are also regions of high tensile strains perpendicular to the load axis which does not coincide with the fillet boundaries. This can apparently be caused by three-dimensional effects like subsurface cracks or cloth layers beneath the surface with reduced bonding between the fillets. Therefore the deformation field in thickness direction was studied with the purpose of illuminating the interaction between the cloth layers during the tensile test. Upon loading, there are visible local displacements which cause high transverse strains in the vicinity of the microstructural feature.

For getting a better understanding of the three-dimensional-effects, the microstructure is analyzed by the use of a micro-CT (micro-computed tomography, Fig. 2). 